\title{
A statistical shape model of the human second cervical vertebra
}

\author{
Marine Clogenson · John M. Duff • Marcel Luethi • \\ Marc Levivier - Reto Meuli - Charles Baur • \\ Simon Henein
}

Received: 20 March 2014 / Accepted: 17 September 2014 / Published online: 30 October 2014

(C) CARS 2014

\begin{abstract}
Purpose Statistical shape and appearance models play an important role in reducing the segmentation processing time of a vertebra and in improving results for 3D model development. Here, we describe the different steps in generating a statistical shape model (SSM) of the second cervical vertebra (C2) and provide the shape model for general use by the scientific community. The main difficulties in its construction are the morphological complexity of the C2 and its variability in the population.

Methods The input dataset is composed of manually segmented anonymized patient computerized tomography (CT) scans. The alignment of the different datasets is done with the procrustes alignment on surface models, and then, the registration is cast as a model-fitting problem using a Gaussian process. A principal component analysis (PCA)-based model is generated which includes the variability of the $\mathrm{C} 2$.

Results The SSM was generated using 92 CT scans. The resulting SSM was evaluated for specificity, compactness and
\end{abstract}

M. Clogenson $(\varangle) \cdot$ C. Baur $\cdot$ S. Henein

École Polytechnique Fédérale de Lausanne, Instant-Lab,

Rue de la Maladière 71b, 2000 Neuchâtel, Switzerland

e-mail: marine.clogenson@epfl.ch

J. M. Duff · M. Levivier

Neurochirurgie, Centre Hospitalier Universitaire

Vaudois et Université de Lausanne, Rue de Bugnon 46,

1011 Lausanne, Switzerland

R. Meuli

Service de radiodiagnostic et radiologie interventionnelle, Centre Hospitalier Universitaire Vaudois et Université de Lausanne, Rue de Bugnon 46, 1011 Lausanne, Switzerland

M. Luethi

Computer Science Department, University of Basel, Bernoullistrasse 16, 4056 Basel, Switzerland generalization ability. The SSM of the C2 is freely available to the scientific community in Slicer (an open source software for image analysis and scientific visualization) with a module created to visualize the SSM using Statismo, a framework for statistical shape modeling.

Conclusion The SSM of the vertebra allows the shape variability of the C2 to be represented. Moreover, the SSM will enable semi-automatic segmentation and 3D model generation of the vertebra, which would greatly benefit surgery planning.

Keywords Statistical shape model - Second cervical vertebra · Non-rigid image registration - Segmentation . Principal component analysis

\section{Introduction}

The increased use of image guided spinal surgery has largely improved precision for implant placement [1-8]. While widely used in the lumbar spine, precision requirements are far more critical in the cervical spine and need optimizing for it's increased use [1]. For example, the target bone volume is much smaller [9], vertebral arteries have a complex and convoluted anatomic relationship to the vertebral bone complex, and the cervical spinal cord and nerve roots are in close proximity [10-13]. Screw implantation in the cervical spine is considered to be high risk [14]: screws are placed in the isthmus, which measures about $6 \mathrm{~mm}$ while the screw diameter is $4 \mathrm{~mm}$ so ideally the implant placement precision error should not exceed $\pm 1 \mathrm{~mm}$. Indeed, overall screw misplacement rates have been well documented [15-17]. The C2, also named axis, presents very specific challenges to the surgeon. Not only is it morphologically the most complex of the entire 
vertebral column, but multiplanar CT 2D preoperative evaluation of this trigonometrically complex 3D structure has not substantially improved screw misplacement rates.

As described in [18], 3D models of an organ or bone can be useful for the surgeon, particularly when bone quality may be poor (for example in osteoporosis), and is not well visualized on standard imaging. This technique may also have a particular application when a part of the $\mathrm{C} 2$ vertebra is affected by a bone destructive pathology (e.g., tumor) and that portion of the vertebra is not fully visualized yet needs to be instrumented. 3D models are generated from segmented CT scans of patients. Image segmentation is the process of partitioning images into different regions that represent anatomic structures. In the above study [18], segmentation of the vertebra is done manually with the 3D Slicer software $[19,20]$. The process takes about $2 \mathrm{~h}$ per vertebra, and this time delay makes it unsuitable for some procedures, especially emergency surgeries. There is a need to automate segmentation processing, and this is the main challenge for computer-assisted medical applications. Major difficulties in image segmentation are the complexity and anatomic variability as well as the image quality available. A complete overview of the different segmentation methods can be found in [21]. In [22], for example, a level set method is used for segmentation but does not incorporate shape prior information. Shape prior segmentation has been the most utilized and is therefore established in medical imaging. The method uses previously obtained data that document morphologic variations of the "standard" shape. This provides anatomic "correction" which can compensate for any image artifacts. A graph cut method considering shape prior information is employed in [23] but does not take into account the shape variation, only the mean of the population shape. Statistical models include shape variations and can therefore be employed to enhance segmentation of human anatomic structures (bones or organs). Probably the best known statistical model algorithm for segmentation is the active shape model [24].

A statistical model (SM) is a model of an anatomic structure that captures the anatomic variability in a sample population obtained from a databank of medical imaging. A SM can be placed into two different categories: The first is the statistical shape model (SSM), which describes the average shape and main variation modes of the bones. The second is the statistical appearance model (SAM), which characterizes the average bone density and variation. In [25], a review discusses the SSM and SAM concepts and their main applications. The authors demonstrate the potential of the SM for diagnosis, evaluation and treatment of skeletal diseases in particular osteoporosis, as well as implant design and surgery planning. Currently, SMs have been created for various anatomic structures and used to segment them. A complete overview of the different SSMs used in medical image segmentation is presented in [26].

One of the main problems in building a SSM is to find correspondence between the reference and target objects in an automatic or semi-automatic way. The objects can be represented by images, surface meshes and point sets obtained from X-ray (2D) or CT scans and MRI (3D); the representation is where the SSM building methods differ. Based on 2D images [27], describes a process to reconstruct 3D lumbar vertebra and in [28], correspondences are found by converting the surfaces into a spherical harmonic descriptions (SPHARM-PDM). However, this method requires closed surfaces. A spectral-based mesh point matching method is used in [29] to register the vertebra followed by statistical shape modeling and a machine learning-based boundary detector for the segmentation while [30] proposes a modelbased segmentation approach using a region-based appearance model with the following different steps: the spine curve extraction, vertebra detection, identification and segmentation. Heitz et al. [31] presents a non-rigid deformation-based method for constructing a SSM of cervical vertebras 6 and 7 (C6 and C7). Group-wise registration as in [32] avoids biasing the SSM to the arbitrarily chosen reference shape. Moreover, a comparison of different registration methods for building SSMs was done for a vertebra and hippocampus (less variability than the vertebra) and showed that in case of bones with high variation in populations, the SSMs are less accurate.

In this study, we examine the feasibility of creating a SSM of the second cervical vertebra. Most of the above methods are for lumbar [27,32] and thoracic vertebra [22,23] or the entire spine [29,30], and only a few studies [31] have considered cervical vertebra, especially the $\mathrm{C} 2$ that is morphologically the most complex. Indeed, this vertebra has a unique and unusual shape to allow efficient head rotation. Certainly, the main difficulties for building the $\mathrm{C} 2$ vertebra SSM and segmenting are its large variability (see Fig. 1), with about $20 \%$ inter-population variation [33] and the difficulty in distinguishing the separation between vertebrae $\mathrm{C} 1-\mathrm{C} 2$ and $\mathrm{C} 2-\mathrm{C} 3$, even directly viewing the $\mathrm{CT}$ scan. But also, the limited surgical exposure and small size of the $\mathrm{C} 2$ vertebra leave few visible identifiable anatomic landmarks, making surface matching a surgical challenge and potential source of error.

In this article, the process of building a SSM of the second cervical vertebra is described in the "Methods" section. The principal characteristic of our model is the use of a Gaussian Process method to perform the registration step. Landmarks are defined to constrain the Gaussian model [34] and so incorporate additional prior information to improve the registration performance and deal with the several shapes of the $\mathrm{C} 2$. A multi-scale Gaussian kernel [35] replaces the Gaussian kernel used in [36] which enforces smoothness and allows 
Fig. 1 Example of three $\mathrm{C} 2$ vertebra

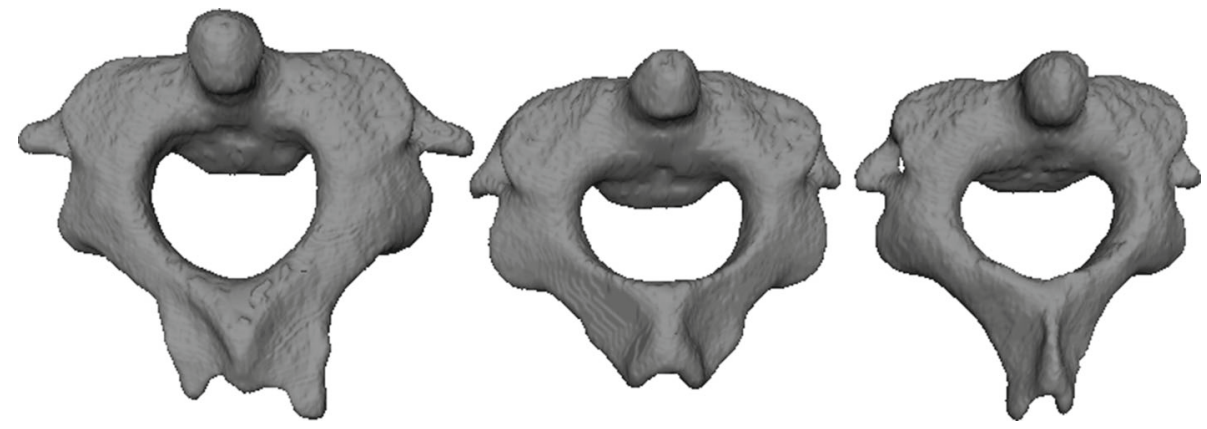

flexible representation of the vertebra for solving the variability problem of the C2. Evaluation of our SSM by measuring of generalization, specificity and compactness [37] and also generating a surface mesh vertebra from a new CT scan is presented in the "Results" section, together with a newly created module and its workflow that allows any SSM to be visualized in the 3D Slicer Software. Having access to a C2 SSM will allow us to semi-automate and speed up the C2 segmentation process and perform rapid surgical planning and/or the surgical planning updates in the operating room, a key issue for surgeon acceptance.

\section{Methods}

The different steps involved in generating a SSM of the C2 are developed in this section and illustrated in Fig. 2.

First, segmentation is performed manually on the input CT scans from the database. Secondly, for vertebra registration,

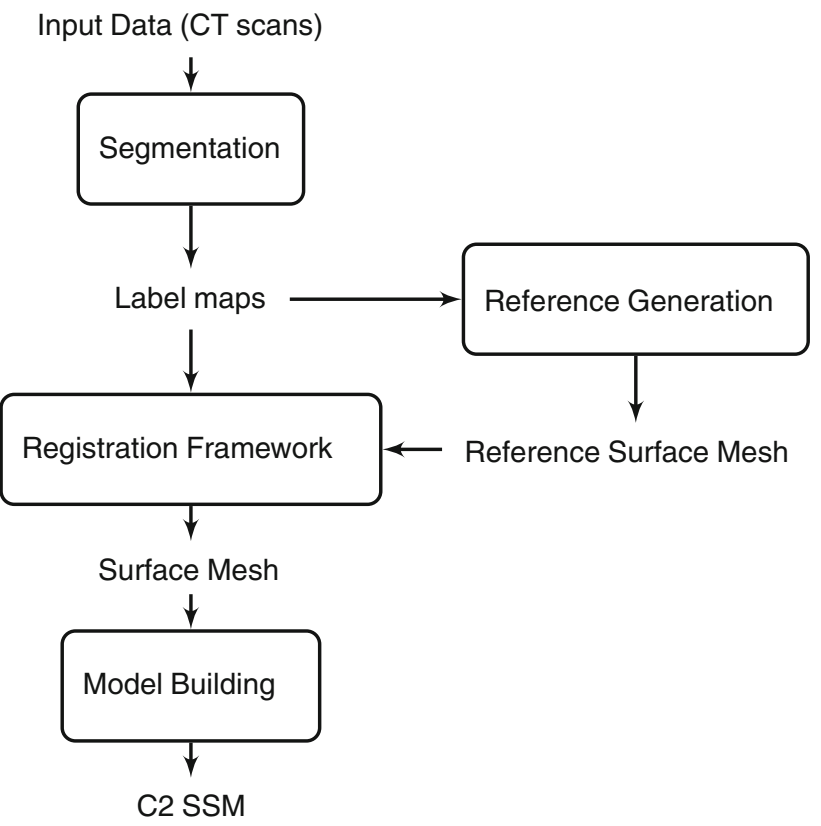

Fig. 2 Flow diagram of the SSM construction alignment of the data is done, thus establishing correlation between the Reference Surface Mesh vertebra and the target vertebrae represented by Label Map. The C2 SSM is built describing the main shape variations of the shapes in the input database anatomy. Mathematically, it refers to an estimate of the probability distribution from the dataset.

Input data

Our input data are composed of a series of 92 anonymized cervical spine CT scans with no C2 pathology (the indication for the CT scans was a history of neck pain in the context of trauma), which meets the requirement of data to represent the shape variations of the object we wish to model. This database has been made available on the virtual skeleton database (VSD) Web site [38], an open access repository for Biomedical Research and Collaboration. The dataset is composed of 42 women and 50 men with an average age of $28.5 \pm 5.8$ years. CT imaging resolution ranged from $0.3 \mathrm{~mm} \times 0.3 \mathrm{~mm} \times 0.6 \mathrm{~mm}$ to $0.3 \mathrm{~mm} \times 0.3 \mathrm{~mm} \times$ $1 \mathrm{~mm}$ spacing. The vertebrae size ranges from $46.75 \mathrm{~mm} \times$ $43.07 \mathrm{~mm} \times 33 \mathrm{~mm}$ to $68.28 \mathrm{~mm} \times 60.32 \mathrm{~mm} \times 56.4 \mathrm{~mm}$.

Segmentation

As a SSM is based on the shape of the organ, only the boundary of the object is useful. Segmentation consists of separating the object from the background to obtain a representation of the surface. It is performed in two steps using the 3D Slicer software $[19,20]$. First, a threshold method is applied to identify the edge of the bone (Fig. 3a). Secondly, a slice-by-slice manual segmentation is necessary to fill the holes and remove the remaining soft tissue (Fig. 3b). The resulting segmentation is called label map and is used in the next SSM building steps.

From the label maps, it is possible to generate a surface mesh vertebra, which is considered as the ground truth and is used to compare and validate the registration method. To obtain the "ideal" vertebra removing small artifacts, the segmentation is smoothed out using the label map smoothing Slicer module with $\sigma=0.5$ (standard deviation of the 
Fig. 3 The different steps required to generate the $\mathrm{C} 2$ surface mesh. a Threshold segmentation. b Slice-by-slice segmentation. c Surface mesh

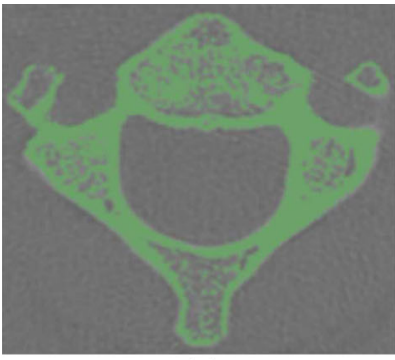

(a)

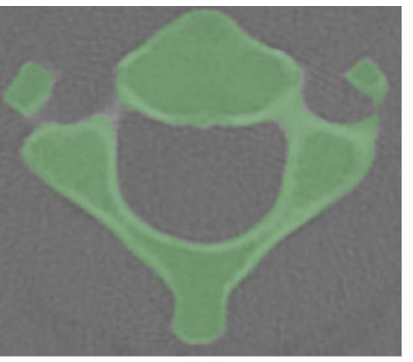

(b)

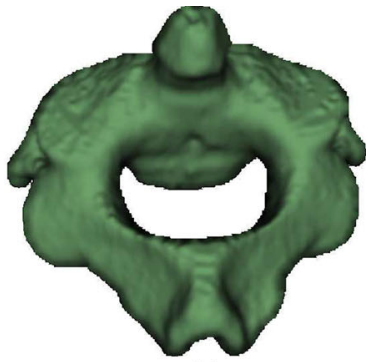

(c)

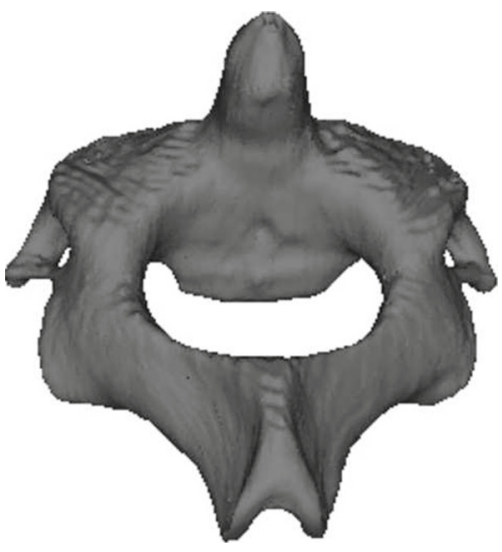

Fig. 4 Reference Vertebra generated from the dataset

Gaussian kernel), resulting in a C2 surface mesh representation (Fig. 3c).

\section{Reference generation}

A surface mesh vertebra used as "reference" for alignment and registration is needed to generate the SSM. To avoid biasing the SSM to a particular vertebra in the training set, a template surface mesh vertebra is generated in an iterative process. A surface mesh vertebra is selected from the dataset and registered to all other label map vertebrae with our method described below (registration framework). The mean is computed and registered to all the training set and a new mean vertebra is produced which is compared to the mean of the previous one. This method is repeated until there is no significant change in the generated vertebra mean. The reference surface mesh vertebra is represented in Fig. 4.

\section{Registration framework}

Registration needs to establish correlation between two objects: in our case between the reference surface mesh and the target image. This is generally the most difficult step in SSM building. The goal is to find a spatial transform $T$, defined as $T(x): \Omega_{\text {ref }} \rightarrow \Omega_{\text {target }}$, where $\Omega_{\text {ref }}$ is the refer-

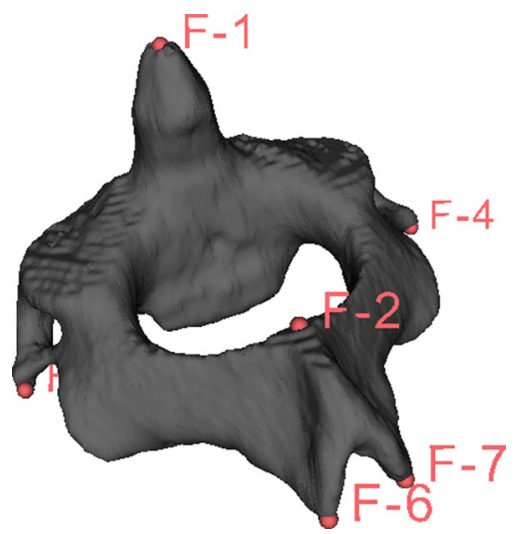

Fig. 5 Surface mesh vertebra with seven landmarks used for alignment

ence object domain and $\Omega_{\text {target }}$ the one of the target objects, which maps one object domain to the other.

Alignment of the vertebrae is performed to initialize the transformation and then a non-rigid registration using a Gaussian Process is applied. So, the transformation $T$ can be seen as a two-step process: a global one, $T_{\text {global }}$ (alignment) and a local one, $T_{\text {local }}$ (non-rigid registration). The advantage of first aligning the dataset is to improve registration results.

\section{Alignment}

A "Procrustes Alignment" (also called landmark registration) is performed to align the dataset to the reference surface mesh. This method consists of defining some landmark points on each image and testing a transformation $\Phi(x)$, which moves the images to match the reference mesh vertebra. The most readily identifiable landmarks are first placed on the Reference vertebra and input data. Figure 5 shows an example of a surface mesh vertebra (for a better overview) marked with seven landmarks as placed in our study.

The transformation is defined as

$\Phi(x)=A x+t$

where $A$ is the rotation matrix, $t$ is a translation vector. The goal is to find a transformation, which minimizes the 
distance between the transformed landmarks of the target object $\left\{\Phi\left(x_{1}\right), \ldots, \Phi\left(x_{m}\right)\right\}$ and the landmarks of the reference shape $\left\{y_{1}, \ldots, y_{m}\right\}$. This is represented mathematically by the following equation:

$$
\begin{aligned}
(A, t) & =\underset{A, t}{\arg \min } \sum_{i=1}^{m}\left\|\Phi\left(x_{i}\right)-y_{i}\right\|^{2} \\
& =\underset{A, t}{\arg \min } \sum_{i=1}^{m}\left\|A\left(x_{i}\right)+t-y_{i}\right\|^{2}
\end{aligned}
$$

The details to solve the equation and its minimum solution are detailed in [39].

\section{Registration}

With the aligned dataset, a point set to image registration is performed to find the spatial transform $u$ that maps points from one surface $\Gamma_{R}$ (the reference surface mesh) to homologous points on the vertebra of the second image $I_{T}$ (the target image) from a space of deformation, $F$. Mathematically, it is defined as the optimization problem:

$$
u=\underset{u \in F}{\arg \min } \mathscr{D}\left[\Gamma_{R}, I_{T}, u\right]+\eta \mathscr{R}[u]
$$

where $\mathscr{D}$ represents a similarity measure and $\mathscr{R}$ a regularizer.

The method for doing this and used in this article is based on [36] where the non-rigid registration is formulated as a model-fitting problem using a Gaussian Process prior that models smooth deformations of the reference vertebra. The model is fitted to the target shape, to obtain a representation of the target shape, which is in correspondence with the reference vertebra.

A Gaussian process is a probability distribution over function $y(x)$ such as the vector $y$ of $y(x)$, evaluated at an arbitrary finite set of points $x_{1}, \ldots, x_{n}$, is Gaussian distributed [40]. A Gaussian Process $y \sim G P\left(\mu, \sum\right)$ is then defined by its mean $\mu=E[y(x)]$ the reference shape in our case, and its covariance (also called kernel) at any two values $\sum\left(x_{n}, x_{m}\right)=E\left[y\left(x_{n}\right) y\left(x_{m}\right)\right]$. A low-rank approximation using the Nyström method is performed on the covariance to make the method computationally feasible and approximate a parametric model so that each deformation can be written as

$u(x)=M\left[\alpha_{1}, \ldots, \alpha_{n}\right]=\mu(x)+\sum_{i=1}^{n} \alpha_{i} \lambda_{i} \phi_{i}(x)$

with $\alpha_{i}$ as the deformation parameters, $\lambda_{i}$ as the eigenvalues and $\phi_{i}$ the eigenvectors of the approximation. The covariance function or kernel defines the process behavior and can be arbitrarily chosen. A multi-scale Gaussian kernel $k_{m g}$ is used here and is defined as $k_{m g}\left(x, x^{\prime}\right)=\sum \alpha_{i} \exp \left(\left\|x-x^{\prime} / \sigma_{i}^{2}\right\|^{2}\right)$ where $\alpha$ is a scale factor. This kernel is utilized to enforce smoothness and also a flexible representation of the vertebra.

Another important addition is the introduction of landmarks placed by a knowledgeable expert in anatomy (radiologist, surgeon). Indeed [34], described a method to use landmarks as additional information by integrating them into the deformation prior.

The deformation of the Gaussian shape model is constrained by the landmark points, which remain fixed. Figure 6 shows the first main variation of the Gaussian Shape Model constrained with the landmarks of the input vertebra.

This constrained shape model is fitted to the distance map (Danielson distance Map in [41]) of the input vertebra label map instead of the label map itself to reduce computational overhead. The advantage of introducing geometric constraints in a shape model is to allow space reduction search in fitting which leads to a more robust registration.

Using that, the deformation $u$ can be expressed as Eq. 4, and introducing the regularization term $\mathscr{R}[u]=$ $\sum_{i=1}^{n} \alpha_{i}^{2} / \lambda_{i}^{2}$, we can formulate the general registration problem (Eq. 3) as

$$
\begin{aligned}
u^{*}= & \underset{\alpha_{1}, \ldots, \alpha_{n}}{\arg \min } \sum_{x_{j} \in \Gamma_{R}} D\left(x_{j}+M\left[\alpha_{1}, \ldots, \alpha_{n}\right]\left(x_{j}\right)\right)^{2} \\
& +\eta \sum_{i=1}^{n} \alpha_{i}^{2} / \lambda_{i}^{2} .
\end{aligned}
$$

Here, $D$ denotes the distance map that was computed from the input labelmap. Equation 5 is a parametric optimization
Fig. 6 A Constrained Shape Model of the vertebra with seven landmark points fixed

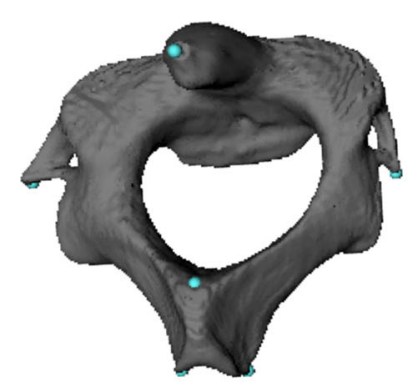

$-2 \sigma$

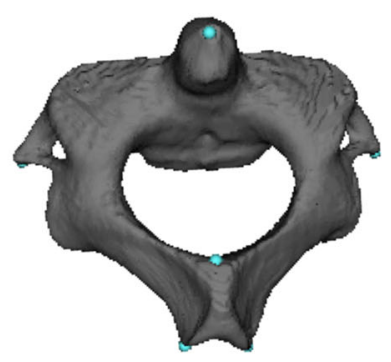

Mean

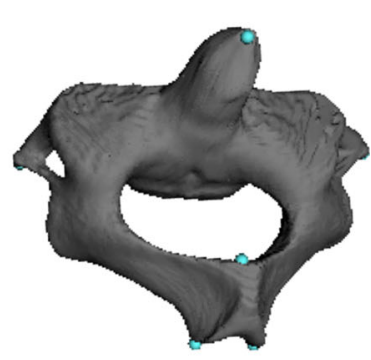

$+2 \sigma$ 


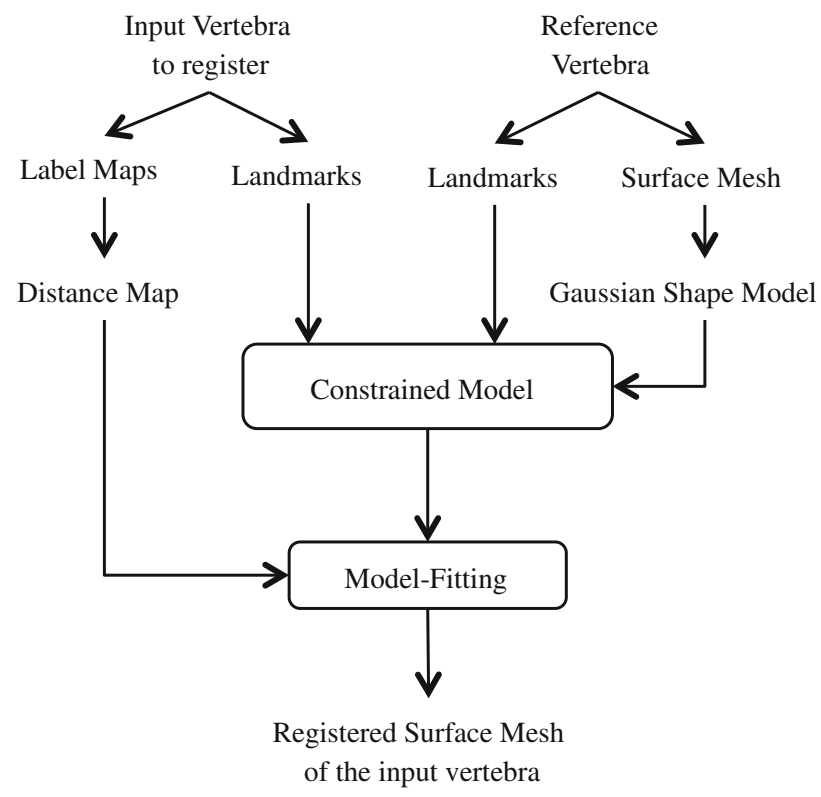

Fig. 7 Flow diagram of the registration method

problem and can be optimizated using a standard optimization approach. We use an LBFGS optimizer, as implemented in ITK [42] and a mean square metric. A summary of the registration steps is presented in Fig. 7.

\section{Model building}

The construction of the SSM is based on a principal component analysis (PCA), which allows the main shape variations to be found. Each surface is represented as a vector and is in correspondence thanks to the registration method. The mean vector $\tilde{v}$ and covariance matrix $S$ are calculated by:

$\tilde{v}=\frac{1}{n} \sum_{i=1}^{n} v_{i}$

$S=\frac{1}{n-1} \sum_{i=1}^{n}\left(v_{i}-\tilde{v}\right)\left(v_{i}-\tilde{v}\right)^{T}$

in which $n$ indicates the number of datasets.

The covariance matrix $S$ can be decomposed as $S=U D^{2} U^{T}$ using a singular value decomposition. The columns $u_{i}$ of the matrix $U$ are the eigenvectors of the covariance matrix $S$, commonly named the principal components of the model, and define the main modes of variation of the dataset. The diagonal matrix $D$ is composed of the corresponding eigenvalues $\lambda_{i}$ of the eigenvectors, which describe the variance of the model represented by the following principal component. The matrix of the eigenvalues $D$ is sorted, so that the first value represents the maximal variance associated with the eigenvector $u_{1}$. In this way, the SSM $v$ can be characterized: $v=v\left(\alpha_{1}, \ldots, \alpha_{m}\right)=\tilde{v}+\sum_{i=1}^{m} \alpha_{i} \lambda_{i} u_{i}$

A new and unique shape is then defined by a vector $\alpha=$ $\left(\alpha_{1}, \ldots, \alpha_{m}\right)$, and $m$ is the number of principal components that model the possible model. The assumption that the vector $\alpha$ is distributed according to a normal distribution $\mathscr{N}\left(0, I_{m}\right)$ leads to the fact that the input objects follow a probability distribution $\mathscr{N}(\tilde{v}, S)$.

Evaluations

Compactness (ability to use a minimal set of parameters), generalization (ability to describe instances outside of the training set) and specificity (ability to represent only valid instances of the object) were used to evaluate the resulting SSM as well generating a surface mesh from a CT scan using the SSM. The open source toolkit Statismo [43] is used to perform registration and model building.

\section{Compactness}

The cumulative variance captured following the number of principal components is calculated. It allows model compactness to be studied that means that the variability of the model must be described using as few modes (or components) as possible. It is calculated as the cumulative variance

$C(M)=\sum_{i=1}^{M} \lambda_{i}$

where $\lambda_{i}$ is the $i$ th eigenvalue. $C(M)$ is measured as a function of the number of principal components $M$. The standard error of $C(M)$ is determined from the number of datasets $n$ :

$\sigma_{C(M)}=\sum_{i=1}^{M} \sqrt{2 / n} \lambda_{i}$

\section{Leave-one-out experiment}

To evaluate the generalized ability of the SSM to represent a new instance of an object (i.e., a new $\mathrm{C} 2$ shape) not present in the training data, a leave-one-out experiment is performed.

A model is created using the reference surface mesh generated in the method section and all of the training samples except one (the test sample). Then the left-out sample is reconstructed using the generated model. To evaluate the error between the test sample and the resulting shape, the root-mean-square (rms) distance is calculated between the two shapes. The experiment is repeated leaving out one training sample each time and an average the distance error calculated. Mathematically, the generalization ability $G(M)$ and its standard error $\sigma_{G(M)}$ are defined as 


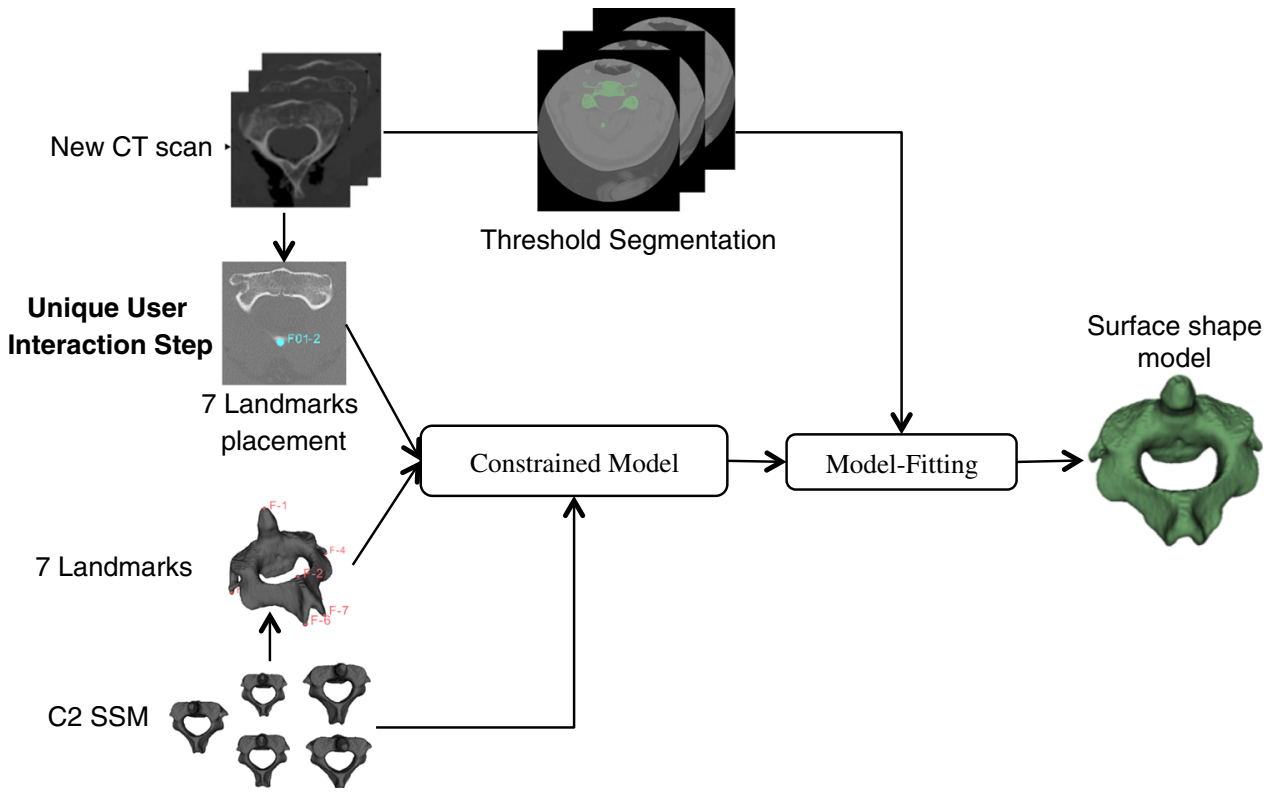

Fig. 8 Flow diagram for generation of a surface shape model from an input CT scan: semi-automatic segmentation

$G(M)=\frac{1}{n} \sum_{i=1}^{n} \mathbb{D}_{i}(M)$

$\sigma_{G(M)}=\frac{\sigma}{\sqrt{(n-1)}}$

where $\mathbb{D}_{i}(M)$ is the rms distance between the two shapes, $n$ the number of samples and $\sigma$ the standard deviation of $G(M)$.

\section{Specificity}

A specific model should only generate instances of the object class that are similar to those in the training set. For this, a population of instances is generated using the SSM and is compared to the data of the training set. The specificity $S(M)$ and its standard error $\sigma_{S(M)}$ are then measured using

$S(M)=\frac{1}{N} \sum_{j=1}^{N} \mathbb{D}_{j}(M)$

$\sigma_{S(M)}=\frac{\sigma}{\sqrt{(} N-1)}$

with $N$ the number of samples ( $N=100$ in our study), $\mathbb{D}_{j}(M)$ the distance between the shape generated by the SSM (in the range of parameters $[-3 \lambda,+3 \lambda]$ ) and the nearest shape of the training set, and $\sigma$ the standard error of $S(M)$.

Using our SSM for the generation of a surface mesh from a CT scan: semi-automatic segmentation

The main application of the SSM that we built above is generating the surface mesh vertebra from the input CT scan allowing, for example, screw placement surgery planning. For this, the method described in the registration part is used with a few adjustments. Automatic threshold segmentation is performed on the input CT scan to obtain a label map. The SSM is then constrained by the landmarks, instead of the Gaussian shape model. The main steps are presented in Fig. 8.

Slicer module (SSM viewer)

A new Slicer module, SSM Viewer was specifically developed to display the created SSM. This module can be downloaded into the latest version of Slicer and installed as an extension.

Thanks to the SSM Viewer module, the main variations in any type of bone can be visualized, in our case, the C2. Figure 9 presents the layout of the module. By varying the sliders of the principal components and the standard deviation, all shape populations are displayed as a surface mesh, and further by converting the surface as a volume in the axial, coronal and sagittal views. The eigen spectrum also appears that enables the number of datasets to be confirmed, very useful for SSM building.

\section{Results and discussion}

Compactness

Shape modes

Figure 10 shows the mean and the first two modes of the PCA. In the first row, the mode corresponding to the largest 


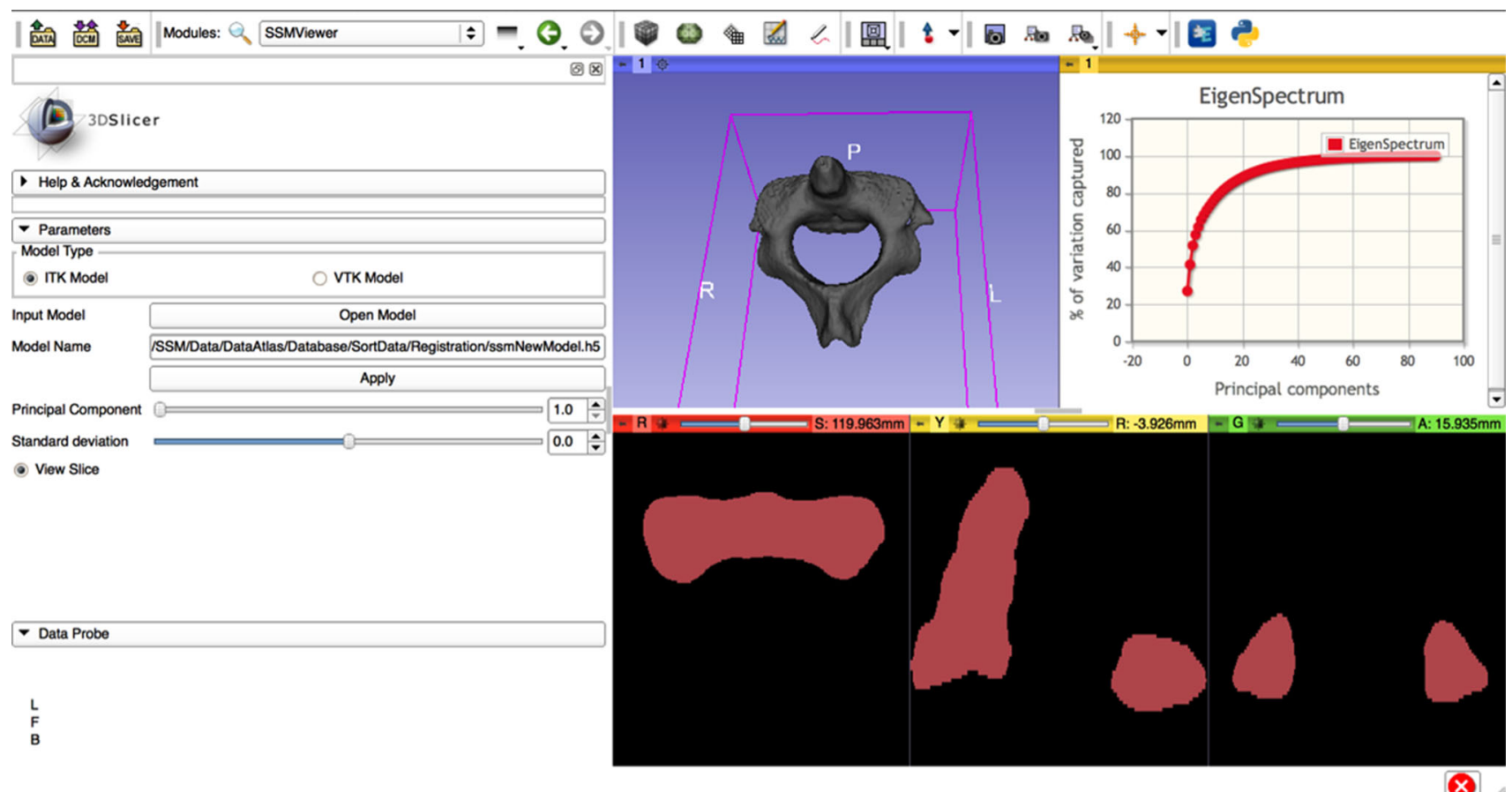

Fig. 9 The Slicer module SSM Viewer to visualize the main shape variations of the model

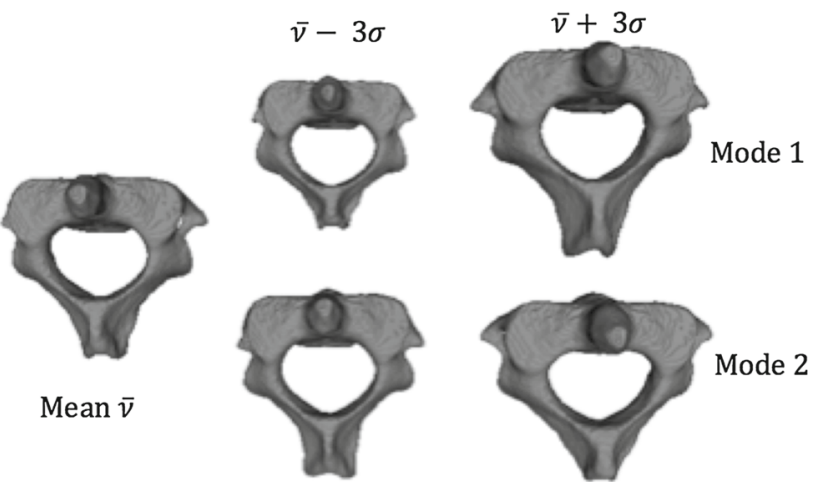

Fig. 10 The first two modes of variation in the linear SSM of the vertebra showing the mean $\pm 3 \mathrm{SD}$

eigenvalue is varied between $-3 \sigma$ and $+3 \sigma$, and in the second row, the same is done for the second mode. The result shows the large variability found in the $\mathrm{C} 2$ and that the size of the bone is mainly captured in the first modes.

\section{Eigen Spectrum}

The cumulative variance captured following the number of principal components is shown in Fig. 11.

The first 20 modes captured $88.5 \%$ of the cumulative variation among the ensemble of the $\mathrm{C} 2$. Specifically, mode 1 captured $27.1 \%$, mode $2,14.2 \%$, mode $3,10.4 \%$, and mode $4,5.7 \%$ of the variation.

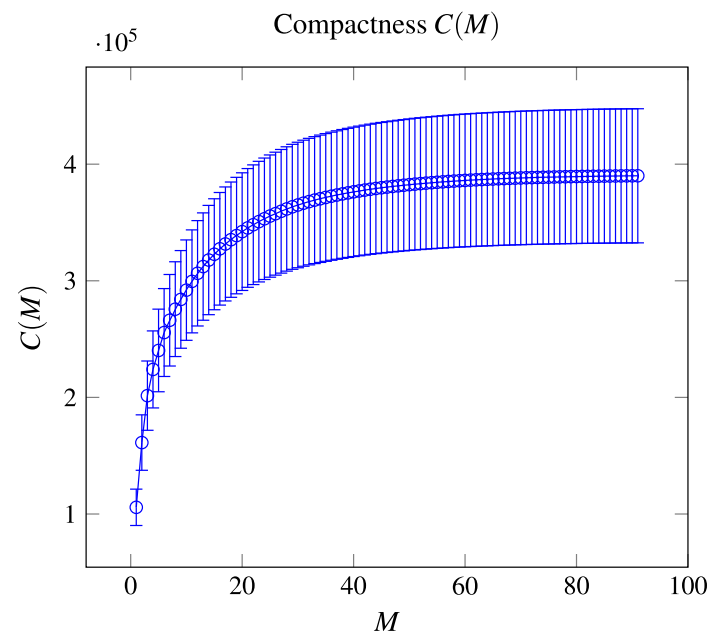

Fig. 11 Captured variance according to the number of principal components

Leave-one-out experiment

The result of the generalization ability $G(M)$ is presented in Fig. 12.

For the first mode of variation, the reconstruction error is $0.93 \mathrm{~mm}$ with a confidence interval of 0.02 while above mode 60 , the error converges on $0.33 \mathrm{~mm}$. The results obtained with our method are better than those presented in [31]. Indeed, for mode 14 , the error is $0.63 \mathrm{~mm}$ with a confidence interval of $1.3 \mathrm{~mm}$ while with our algorithm, it is $0.59 \mathrm{~mm}$ with $0.01 \mathrm{~mm}$ 


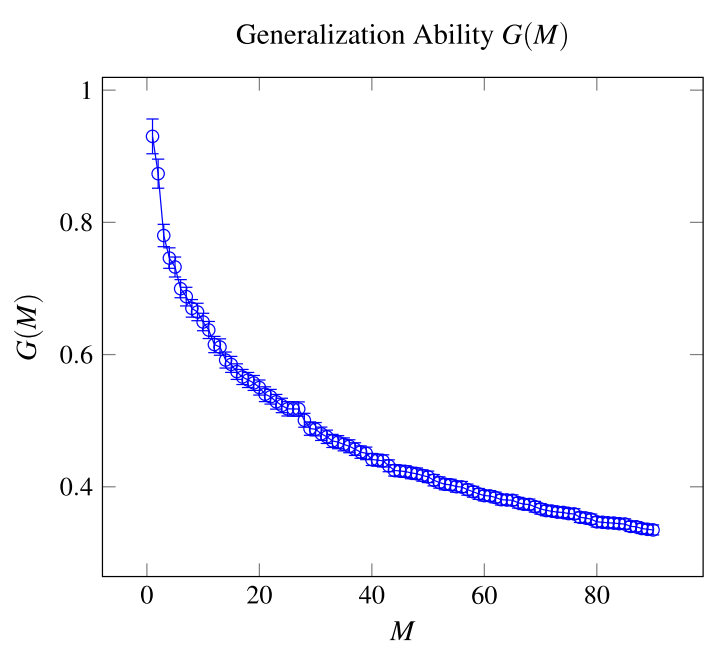

Fig. 12 Leave-one-out experiment following the number of principal components

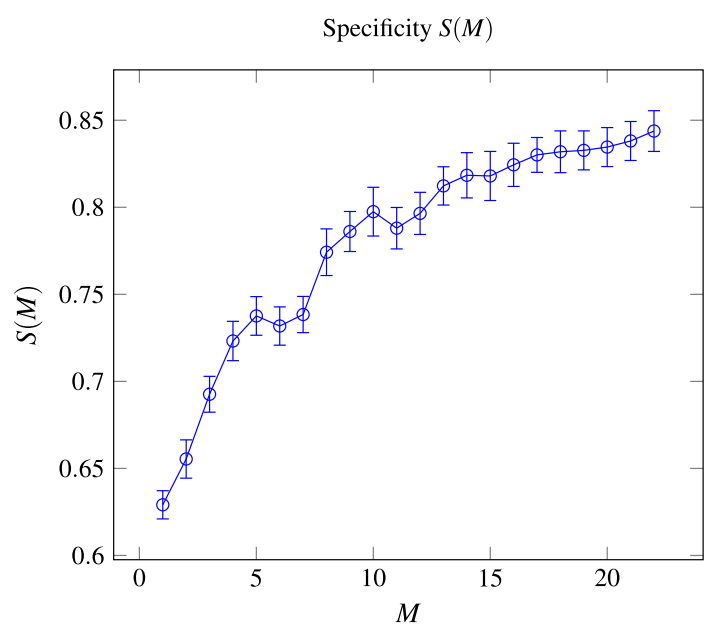

Fig. 13 Specificity following the number of principal component

of SE. Moreover, in [32], for L2 vertebra, four methods are compared and generalization with rms distance converges with values above $0.7 \mathrm{~mm}$ which is larger than the values obtained here.

\section{Specificity}

The result for the specificity is shown in Fig. 13. Our model shows better specificity than the models presented in [32], which is important to represent only valid instances of the vertebra. Indeed, for mode 10 , our specificity is about 0.8 instead of more than 1.2 for others.

Generation of a surface mesh from a CT scan

C2 Surface mesh generation was tested on $31 \mathrm{CT}$ scans from anonymous patients. Using the 3D Slicer software [20] (manual segmentation), generation of a representative surface

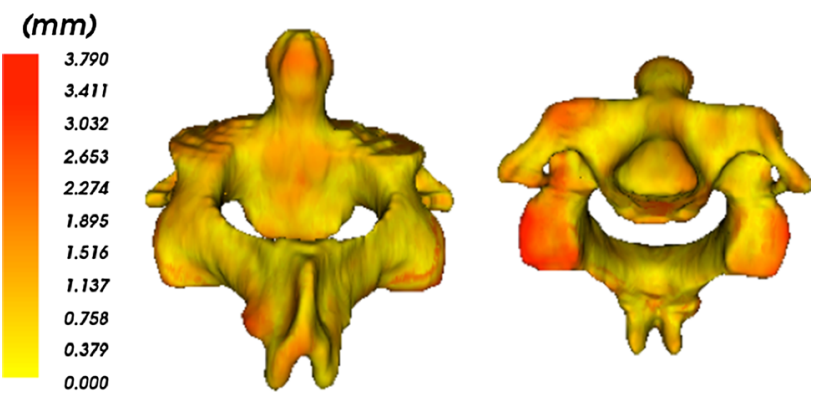

Fig. 14 Euclidean Distance between the manual and generated surface mesh vertebra

Table 1 Comparison with different methods

\begin{tabular}{llll}
\hline & {$[29]$} & {$[30]$} & Our method \\
\hline Mean $(\mathrm{mm})$ & 1.4 & 0.81 & 0.9 \\
$\mathrm{SD}(\mathrm{mm})$ & 0.4 & 0.97 & 0.12 \\
Segmentation time & $2 \mathrm{~min}$ & $3 \mathrm{~min}$ & $30 \mathrm{~s}$ \\
\hline
\end{tabular}

mesh takes about $1 \mathrm{~h}$. In contrast, using our SSM method, an average of $2 \mathrm{~min}$ is required from landmark placement on the CT scans to surface mesh generation. The Euclidean distance from the manually generated surface shape and that obtained using our segmentation algorithm gives a mean distance error of $0.90 \pm 0.12 \mathrm{~mm}$. The shape differences between the manual and SSM-generated surface mesh are mainly located in the inferior articular facet of the vertebra (see Fig. 14), a zone where the distinction between two vertebras is difficult to identify even directly viewing the CT scan.

We compared our method to the methods used in [30] and [29] which also use Statistical Models for vertebra segmentation. Table 1 presents the results for the mean and the standard deviation of the distance error between the ground truth mesh (manual segmentation) and the generated mesh, and also the computation time of the $\mathrm{C} 2$ vertebra algorithm.

Our method has a slightly higher mean error than [30] but is better than [29]. Moreover compared to both, the standard deviation is lower with our method. Our SSM-based segmentation is clearly faster than the other two methods ( $30 \mathrm{~s}$ vs. 2 and $3 \mathrm{~min}$ ), not considering landmark placement by the user. If we take into account landmark placement, segmentation takes $2 \mathrm{~min}$, comparable to the other techniques, which are entirely automatic (no landmarks). While we loose a bit of speed and the automatic of the algorithm we gain in precision of the segmentation of the $\mathrm{C} 2$.

The SSM robustness in segmentation could be improved by focusing the landmarks to the region of interest depending on the problem: the isthmus for screw placement for example. Fitting can also be enforced in particular zones such as the inferior articular facet, to get closer to the manually segmented vertebra. 


\section{Conclusion}

A SSM of the second cervical vertebra was generated from CT scans of patients with normal C2 vertebrae and clearly illustrates its anatomic variability. One of the major challenges was to find point-to-point correspondence between the reference surface mesh vertebra and the input data vertebrae represented as Label Maps. This was resolved using a Procrustes Alignment" on the manually defined landmarks to align the Label Maps and registration is treated as a model-fitting problem. In generating the SSM a Gaussian Process method is used for registration and the incorporation of additional prior information by defining landmarks to constrain the Gaussian shape model. The novelty of this method is a multi-scale Gaussian kernel for the Gaussian Process that replaces the usual simple Gaussian kernel and is utilized to enforce smoothness as well as a flexible representation of the vertebra, necessary for its high variability.

Compared to state-of-the art methods for SSM building, this registration method presents an improvement in results for a complex and variable bone like $\mathrm{C} 2$. The compactness analysis with the eigen spectrum shows that this registration method allows compact SSM to be obtained. Similarly, generalization demonstrates that the SSM can represent a new unknown vertebra shape and outperform the results obtained with other SSMs. The specificity analysis presented better results, which is important to represent only valid instances of the vertebra. The first results for the SSM robustness in segmentation show clear improvements compared to previous methods due to incorporation of prior information though user interaction is needed.

In conclusion, SSMs are invaluable tools for rapid and precise surface merge algorithms for 3D model generation, diagnosis (scoliosis, trauma modeling of broken vertebrae elements) and robotic surgeries.

Notably, using the C2 SSM described here, the surgeon will obtain the 3D model of the vertebra almost instantaneously after the patient CT scan, enabling rapid planning or adaptation of planning for spine surgery. This process will facilitate image guidance surgical techniques for complex $\mathrm{C} 2$ instrumentation for segmental vertebral instability due to trauma, destructive tumors or inflammatory processes. Additionally our SSM may be extended for use in a wide range of bone or organ surgeries.

Acknowledgments The Swiss National Science Foundation (SNSF) supported this study. The authors thank KB Medical for their help and support with the project.

Conflict of interest The authors declare that they have no conflict of interest.

\section{References}

1. Ishikawa Y, Kanemura T, Yoshida G, Ito Z, Muramoto A, Ohno S (2010) Clinical accuracy of three-dimensional fluoroscopy-based computer-assisted cervical pedicle screw placement: a retrospective comparative study of conventional versus computer-assisted cervical pedicle screw placement. J Neurosurg Spine 13(5):606611

2. Ito H, Neo M, Yoshida M, Fujibayashi S, Yoshitomi H, Nakamura $\mathrm{T}$ (2007) Efficacy of computer-assisted pedicle screw insertion for cervical instability in RA patients. Rheumatol Int 27(6):567-574

3. Kamimura M, Ebara S, Itoh H, Tateiwa Y, Kinoshita T, Takaoka K (2000) Cervical pedicle screw insertion: assessment of safety and accuracy with computer-assisted image guidance. J Spinal Disord 13(3):218-224

4. Ludwig SC, Kramer DL, Balderston RA, Vaccaro AR, Foley KF, Albert TJ (2000) Placement of pedicle screws in the human cadaveric cervical spine: comparative accuracy of three techniques. Spine 25(13):1655-1667

5. Rampersaud YR, Pik JHT, Salonen D, Farooq S (2005) Clinical accuracy of fluoroscopic computer-assisted pedicle screw fixation: a CT analysis. Spine 30(7):E183-190

6. Richter M, Cakir B, Schmidt R (2005) Cervical pedicle screws: conventional versus computer-assisted placement of cannulated screws. Spine 30(20):2280-2287

7. Richter M, Mattes T, Cakir B (2004) Computer-assisted posterior instrumentation of the cervical and cervico-thoracic spine. Eur Spine J 13(1):50-59

8. Weidner A, Wähler M, Chiu ST, Ullrich CG (2000) Modification of c1-c2 transarticular screw fixation by image-guided surgery. Spine 25(20):2668-2673, discussion 2674

9. Panjabi MM, Shin EK, Chen NC, Wang JL (2000) Internal morphology of human cervical pedicles. Spine 25(10):1197-1205

10. Karaikovic EE, Daubs MD, Madsen RW, Gaines JRW (1997) Morphologic characteristics of human cervical pedicles. Spine 22(5):493-500

11. Karaikovic EE, Yingsakmongkol W, Gaines JRW (2001) Accuracy of cervical pedicle screw placement using the funnel technique. Spine 26(22):2456-2462

12. Neo M, Fujibayashi S, Miyata M, Takemoto M, Nakamura T (2008) Vertebral artery injury during cervical spine surgery: a survey of more than 5600 operations. Spine 33(7):779-785

13. Neo M, Sakamoto T, Fujibayashi S, Nakamura T (2005) The clinical risk of vertebral artery injury from cervical pedicle screws inserted in degenerative vertebrae. Spine 30(24):2800-2805

14. Roy-Camille R, Saillant G, Laville C, Benazet JP (1992) Treatment of lower cervical spinal injuries-c3 to c7. Spine 17(10 Suppl):S442-446

15. Kosmopoulos V, Schizas C (2007) Pedicle screw placement accuracy: a meta-analysis. Spine 32(3):E111-120

16. Tian NF, Xu HZ (2009) Image-guided pedicle screw insertion accuracy: a meta-analysis. Int Orthop 33(4):895-903

17. Verma R, Krishan S, Haendlmayer K, Mohsen A (2010) Functional outcome of computer-assisted spinal pedicle screw placement: a systematic review and meta-analysis of 23 studies including 5,992 pedicle screws. Eur Spine J 19(3):370-375

18. Kostrzewski S, Duff JM, Baur C, Olszewski M (2012) Robotic system for cervical spine surgery. Int J Med Robot 8(2):184-190

19. Fedorov A, Beichel R, Kalpathy-Cramer J, Finet J, Fillion-Robin JC, Pujol S, Bauer C, Jennings D, Fennessy F, Sonka M, Buatti J, Aylward S, Miller JV, Pieper S, Kikinis R (2012) 3D slicer as an image computing platform for the quantitative imaging network. Magn Reson Imaging 30(9):1323-1341

20. 3D slicer. www.slicer.org/ 
21. Sharma N, Aggarwal LM (2010) Automated medical image segmentation techniques. J Med Phys 35(1):3-14

22. Aslan M, Farag A, Arnold B, Xiang P (2011) Segmentation of vertebrae using level sets with expectation maximization algorithm. In: 2011 IEEE international symposium on biomedical imaging: from nano to macro, pp 2010-2013

23. Aslan M, Ali A, Farag A, Rara H, Arnold B, Xiang P (2010) 3d vertebral body segmentation using shape based graph cuts. In: 2010 20th international conference on pattern recognition (ICPR), pp 3951-3954

24. Cootes TF, Taylor CJ, Cooper DH, Graham J (1995) Active shape models - their training and application. Comput Vis Image Underst 61(1):38-59

25. Sarkalkan N, Weinans H, Zadpoor AA (2014) Statistical shape and appearance models of bones. Bone 60:129-140

26. Heimann T, Meinzer HP (2009) Statistical shape models for 3d medical image segmentation: a review. Med Image Anal 13(4):543-563

27. Zheng G, Nolte LP, Ferguson SJ (2011) Scaled, patient-specific 3D vertebral model reconstruction based on 2D lateral fluoroscopy. Int J Comput Assist Radiol Surg 6(3):351-366

28. Styner M, Oguz I, Xu S, Brechbuhler C, Pantazis D, Levitt JJ, Shenton ME, Gerig G (2006) Framework for the statistical shape analysis of brain structures using SPHARM-PDM. Insight J 1071:242250

29. Mirzaalian H, Wels M, Heimann T, Kelm B, Suehling M (2013) Fast and robust $3 \mathrm{~d}$ vertebra segmentation using statistical shape models. In: 2013 35th annual international conference of the IEEE engineering in medicine and biology society (EMBC), pp 33793382

30. Klinder T, Ostermann J, Ehm M, Franz A, Kneser R, Lorenz C (2009) Automated model-based vertebra detection, identification, and segmentation in CT images. Med Image Anal 13(3):471-482

31. Heitz G, Rohlfing T, Maurer CR Jr (2005) Statistical shape model generation using nonrigid deformation of a template mesh, pp $1411-1421$
32. Rasoulian A, Rohling R, Abolmaesumi P (2012) Group-wise registration of point sets for statistical shape models. IEEE Trans Med Imaging 31(11):2025-2034

33. Madawi AA, Casey AT, Solanki GA, Tuite G, Veres R, Crockard HA (1997) Radiological and anatomical evaluation of the atlantoaxial transarticular screw fixation technique. J Neurosurg 86(6):961-968

34. Lüthi M, Jud C, Vetter T (2011) Using landmarks as a deformation prior for hybrid image registration. In: Proceedings of the 33rd international conference on pattern recognition, Berlin, Heidelberg, DAGM'11, pp 196-205

35. Opfer R (2006) Multiscale kernels. Adv Comput Math 25(4):357380

36. Lüthi M, Jud C, Vetter T (2013) A unified approach to shape model fitting and non-rigid registration. In: Machine learning in medical imaging, no. 8184 in lecture notes in computer science, pp 66-73

37. Styner MA, Rajamani KT, Nolte LP, Zsemlye G, Székely G, Taylor CJ, Davies RH (2003) Evaluation of 3d correspondence methods for model building. In: Taylor C, Noble JA (eds) Information processing in medical imaging, no. 2732 in lecture notes in computer science, pp 63-75

38. Kistler M, Bonaretti S, Pfahrer M, Niklaus R, Buchler P (2013) The virtual skeleton database: An open access repository for biomedical research and collaboration. J Med Internet Res 15(11):e245

39. Umeyama S (1991) Least-squares estimation of transformation parameters between two point patterns. IEEE Trans Pattern Anal Mach Intell 13(4):376-380

40. Rasmussen CE, Williams CKI (2006) Gaussian processes for machine learning. MIT Press, Cambridge, MA

41. Danielsson PE (1980) Euclidean distance mapping. Comput Graph Image Process 14(3):227-248

42. The insight segmentation and registration toolkit. www.itk.org

43. Lüthi M, Albrecht T, Gass T, Goksel O, Kistler M, Bousleiman H, Reyes M, Buechler P, Cattin PC, Vetter T (2012) Statismo-a framework for PCA based statistical models. Insight J 1:1-18 